\title{
An agent-based simulation model of dynamic real-time traffic signal controller
}

\author{
Ahmad Aljaafreh ${ }^{1 *}$, Ma'en Saleh $^{2}$, Naeem Al-Oudat $^{3}$ and Murad Alaqtash ${ }^{2}$ \\ Associate Professor, Department of Communications, Electronics and Computer Engineering, Tafila Technical \\ University, Jordan ${ }^{1}$ \\ Assistant Professor, Department of Electrical and Computer Engineering, Tafila Technical University, Jordan ${ }^{2}$ \\ Faculty Member, Department of Communications and Computer engineering, Tafila Technical University, Jordan ${ }^{3}$
}

Received: 02-July-2019; Revised: 05-December-2019; Accepted: 10-December-2019

(C)2020 Ahmad Aljaafreh et al. This is an open access article distributed under the Creative Commons Attribution (CC BY) License, which permits unrestricted use, distribution, and reproduction in any medium, provided the original work is properly cited.

\begin{abstract}
Traffic management has a key role within intelligent transportation systems (ITS). An efficient traffic control system leads to less fuel consumption, gas emissions, and transportation delay. The main goal of this study is to minimize the driver waiting time at intersections and avoid traffic jams. To achieve this goal, an adaptive traffic signal controller model was proposed and verified in this work. A multi-agent based adaptive controller for a dynamic vehicular ad-hoc network (VANET) in a single four-way intersection was modelled and simulated using Matlab/Simulink/SimEvents. The inputs of the controller are the position and the speed of the vehicles at each approach. In fact, the need for inter-vehicle communication is eliminated in this work through the process of virtual road segmentation and the deployment of roadside units (RSUs). The output of the controlled is the green-time for each approach, where the green time is calculated based on the traffic density and on the queue length. The proposed model was verified by intensive simulations using four main parameters: green phase time; inter-arrival times; service time; and average waiting time (AWT). The performance, $A W T$, of the proposed adaptive controller was compared to a fixed-time controller. The simulation results showed that the proposed controller outperformed the fixed-time controller for significant variance (i.e. variance > 10) in the means of inter-arrival times for vehicles approaching the traffic signal.
\end{abstract}

\section{Keywords}

VANETs, Controller, RSU, Traffic management, Agents, Simulink, Intelligent transportation systems (ITS).

\section{Introduction}

Traffic conflict at the intersection is considered one of the main problems of traffic management. Traffic signals are considered a cheap tool for solving this problem through time division multiplexing approach. The delay time at signalized intersections is considered the best performance measure that reflects the efficiency of traffic signal controller. Traffic signal controllers can be classified into conventional fixed-time and real-time adaptive controllers. The most widely used is the fixed-time controller. This type of controller has a fixed and preset cycle time based on prior information about the traffic, where cycle time is the green and clearance intervals for all phases of traffic signal. The fixed-time controller has the benefit of being simple, though it doesn't adapt to changes in traffic conditions and flow variability.

*Author for correspondence

1
An adaptive real-time controller changes cycle time and phase sequence based on the traffic flow and condition. Sensors are used to detect vehicles, embedded sensors in road pavement or camera [1].

Another type of traffic signal controller is the fuzzy controller [2]. Both cycle time and phase sequence are changed dynamically according to traffic density and delays at the intersection, where traffic density is the average number of vehicles in one kilometer of road. A fuzzy rule-based system was used to specify the greenest time of the phase sequence. The simulation results shown that fuzzy controller has better performance than fixed-time controller in heavy traffic conditions [3].

An improved discrete event simulation model of traffic light control at a single intersection was described and validated in [4]. The performance was indicated by the average waiting time, and the 
number of vehicles at each stream. The adaptive time of red/green light was calculated using conflict directions matrix described in [5]. The simulation of fuzzy traffic controller was performed in [6] for multi-lane isolated signalized intersection. A model for an intersection of two lanes and different values for waiting time and queue length was performed for each road segment. The maximum values of waiting time and queue length represented the input of the fuzzy controller.

The main goal of this work is to minimize the driver waiting time at intersections and avoid traffic jams. Indeed, an optimal traffic signal controller can achieve this goal. Traffic signal optimization is one of the most effective methods to reduce traffic congestion [7-13]. The problem can be modeled as an optimization problem, where the objective function is the average waiting time, and the optimization parameters are the green time for each phase. Thus, the goal is to find the best value for these parameters in order to minimize the objective function. However, this problem is hard and blind due to abnormal and random conditions $[14,15]$. Therefore, the objective of this paper is to find the optimal solution for this problem.

Conventional simulation techniques are suitable for fixed-time approaches [16]. However, they are not sufficient for heterogeneous environments such as highly-dynamic topology real-time Vehicular AdHoc Network (VANET) with QoS guarantees [17]. To overcome the limitations of conventional simulation techniques, agent-based simulation techniques were used to model and analyze such dynamic environments, where the whole system is decomposed into a set of cooperative sub-agents with a well-defined communication protocol between them [17-19].

Most of the above-mentioned adaptive systems are based on the queue length and/or traffic flow on the intersection approaches. Although these systems consider the present situation of the traffic, the future state is neglected. The future status of the traffic situation is denoted by traffic density which represents the number of vehicles in one-kilometer length of the street. Traffic density can be calculated from the position and the speed of approaching vehicles which can be obtained by utilizing the intervehicle communication.

In this paper, a multi-agent system for dynamic realtime traffic signal controller was modelled using 2
Matlab/Simulink/SimEvents and compared to other controllers. The inputs of the controller are the position and the speed of vehicles at each road, based on the assumption that data communication is possible between traffic signal systems, vehicles, and roadside units. The output of the controlled is the green-time for each road, where the green time is calculated based on the traffic density and on the queue length. Since every vehicle sends its position and speed, the controller can predict the time of arrival for each vehicle.

The main contribution of this work is as follows:

- The design and simulation of an adaptive controller model for a dynamic real-time VANET in a single four-way intersection using Matlab/Simulink/SimEvents

- The use of agent-based methodology for system design to eliminate the limitations of conventional simulation techniques.

- Eliminating the need of Inter-vehicle communication through the process of virtual road segmentation and the deployment of road-side units (RSUs) for communication.

The rest of this paper is organized as follows. Section 2 presents the agent-based system model. In Section 3 , a discrete simulation model is described in details. Section 4 verifies the simulation model by comparison with fixed time models. The conclusion of this research and future research directions are provided in Section 5.

\section{Methodology and system model}

Using agent-based methodology, three main phases were defined: 1) Decomposition, where the system is decomposed into five sub-agents: vehicle, queue, server, controller, and roadside unit (RSU). 2) Modelling, where the functionality of each sub-agent is defined. 3) Protocol, where the interaction between sub-agents is defined.

\subsection{Sub-agents design}

The decomposition of the agent-based system along with the communication links between agents is shown in Figure 1. The sub-agents of the proposed multi-agent model are defined as follows:

1) Vehicle: This sub-agent has an ID that is the plate number, the MAC address in the communication protocol, as well as a specific speed. An exponential distribution with mean $(1 / y)$ was used to generate the inter-arrival time for the vehicle, while a uniform distribution was used to model its 
speed. Each vehicle has the ability to know its position (coordinates) via GPS signals.

2) Queue: queuing and de-queuing processes are performed by this agent. The arrived vehicle will be queued based on its arrival-time, while the vehicle at the top of the queue is to be de-queued to be served by the server sub-agent. This agent is to be downloaded at the traffic light signal.

3) Server: An exponential function with a mean $(1 / y)$ was used to model the service time of the vehicle, where $(y)$ is the vehicle service rate that is affected by some metrics such as the size of the vehicle, its location in the lane, and the lane dimensions. This agent is to be downloaded at the traffic light signal.

4) Controller: It is the coordinator of the whole process. It collects system information from other sub-agents and evaluates system parameters needed to govern the functionalities of all subagents. Such sub-agent is to be downloaded at the traffic light signal.

5) Roadside unit (RSU): Such sub-agent monitors the virtual road segment. It is the intermediate agent between the controller and the vehicles. Each subagent has a specific ID (SID).

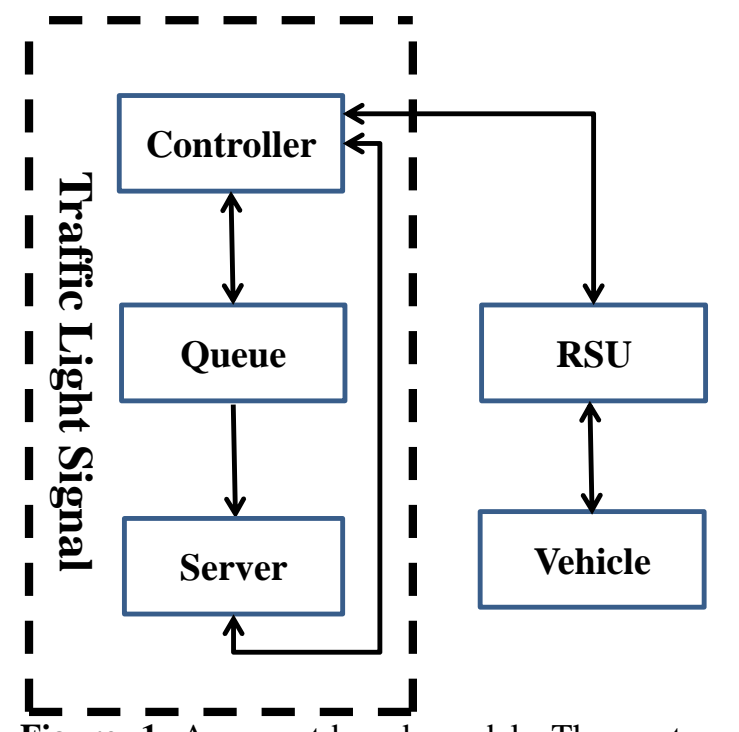

Figure 1 An agent-based model. The system is decomposed into five sub-agents: vehicle, queue, server, controller, and roadside unit (RSU)

\subsection{Assumptions and system parameters}

Figure 2 illustrates the various parts of the overall system. To model the proposed system, assumptions and network parameters should be well defined, i.e. the mobility model of the vehicles, RSUs and node parameters, and communication scheme.

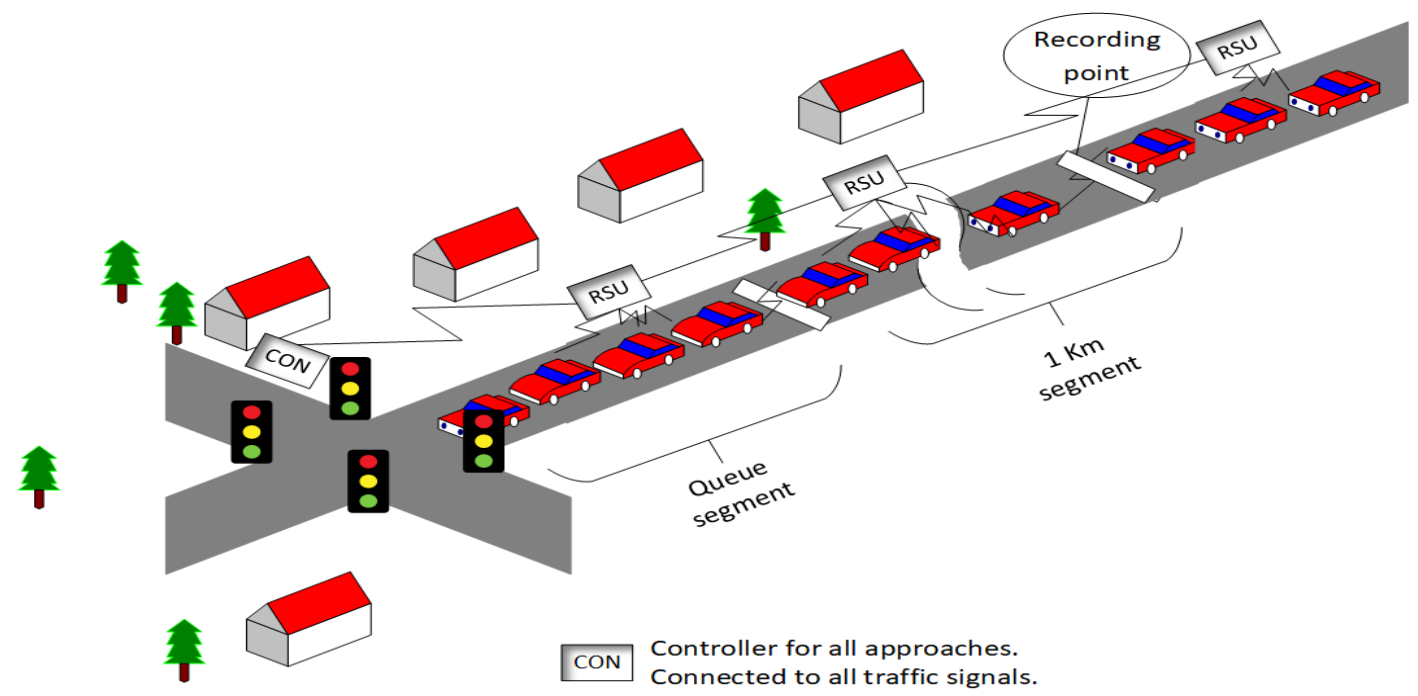

Figure 2 Overall model description at four-way single intersection

\subsubsection{Assumptions}

In this work, the mobility model was defined in the previous work [5], where vehicles are moving in the same direction without changing their speed. The virtual road segment is monitored by the RSU, the transmission range of RSU reaches all vehicles in the road-segment. To ensure proper communication, we assumed that RSU has the ability to communicate with its adjacent RSUs and each node is in the center of its transmission circle. 


\subsubsection{System parameters}

The parameters for both road segment and mobile node (vehicle) are defined as follows:

1) Road segments: $X$ number of road-segments with the following attributes:

a. ID of the segment $\left(S_{I D}\right)$.

b. Segment dimensions $(L, W)$ : $L$ and $W$ are the length and width of the segment.

1) Mobile nodes: $\mathrm{N}$ number of mobile nodes (vehicles) is assumed in each road segment and each node has the following attributes:

a) Node address $\left(N_{I D}\right)$ : Each vehicle node in the segment has a unique address that is the plate number (looks like the MAC address).

b) Road segment where the node belongs $(R)$.

c) Node speed $\left(N_{S}\right)$ : Nodes are moving in the same direction with a uniform distribution between 20 and $60 \mathrm{Km} / \mathrm{h}$.

d) Node position $\left(P_{x}, P_{y}\right)$.

e) Strength of signals received by the node from the RSUs. No more than two signals could be received $\left(\alpha_{1}, \alpha_{2}\right)$. Such parameters are the key behind defining the segments' boundaries.

2.2.3Communication schemes

The communication scheme between RSUs is defined according to the position of the RSU as follows:

1) Intra-segment scheme: That is when the RSU and the traffic light are located in the same segment. In such case, the communication between the RSU and the controller is direct.

2) Inter-segment communication: In such case, the RSU and the traffic light belong to different segments. Accordingly, a layer of cooperation between adjacent RSUs is exists.

\subsection{Sub-agents interaction}

The process begins when the controller sub-agent initiates a system-begin-request, every time $t=T$, and sends it to the RSU that serves the segment where the traffic light exists. Such request will be broadcast to every RSU in the road. Upon receiving such request, each RSU broadcasts a control message in the virtual segment it serves requesting the vehicle nodes to send their system parameters, node position $\left(P_{x}, P_{y}\right)$ and node speed $\left(N_{S}\right)$. Accordingly, each vehicle node responds by sending such parameters, destination address is RSU.

One of the problems in such model is that when a vehicle receives requests from two different RSUs. i.e., the vehicle is an intermediate one. Such problem was solved by embedding a signal strength comparator in the vehicle. Accordingly, the vehicle responds to the strongest one. That is if $\left(\alpha_{1}>\alpha_{2}\right)$ then the vehicle belongs to RSU1.

Upon receiving such vehicles parameters, each RSU sends them to the controller sub-agent. According to the location of the RSU, the communication scheme is following one of the two modes that have been previously mentioned in section 2.2.3. Once the controller receives the parameters, it interacts with the queue sub-agent requesting for its size $(Q)$ that is the number of vehicles in the virtual segment where the traffic light exists. Upon receiving such information, the controller then calculates the traffic density $\left(T D_{x}\right)$ for approach $x$ as:

$T D_{x}=\frac{\sum_{i=1}^{m} N_{i}}{\sum_{i=1}^{m} L_{i}}$

Where $m$ is the number of virtual segments in the approach, $N_{i}$ is the number of vehicles in each virtual segment $i, L_{i}$ is the length of segment $i, Q_{x}$ is the size of the queue in approach $x$. i.e. our system is a single intersection where 4 approaches are exist, $x \in$ $\{1,2,3,4\}$.

Once a traffic density is obtained for approach $x$, the controller calculates the green interval indicator GII for this approach as:

$G I I_{x}=T D_{x}+Q_{x}$

Accordingly, the controller calculates the green time $\left(G_{x}\right)$ for approach $x$ as:

$$
G_{x}=\frac{G I I_{x}}{\sum_{i=1}^{4} G I I_{i}} \times\left(T_{c}-4 T_{y}\right)
$$

where $T_{c}$ is the cycle time, and $T_{y}$ is the yellow time. The controller then evaluates the service time $(S)$ for each vehicle in the queue; given that the queue is applying a First-In-First-Out (FIFO) scheduling algorithm where the vehicle at the top of the queue is the nearest to the traffic light and should be served first. The calculation of the vehicle's service time is as:

$$
S_{i+1}=S_{i}+\lambda e^{-\lambda i}
$$

where $i \in\{1,2,3, \ldots n\}, S_{i}$ is the service time for vehicle number $i$ in the queue, such that the first service time is $S_{1}$. $S_{0}$ is initialized to zero.

Upon evaluating the service time of the vehicles, the controller interacts with queue and requests it to dequeue the vehicle from the top of the queue and passes it to the server. The controller also passes the service time of the de-queued vehicle to the server 
that serves the vehicle in turns. Figure 3 illustrates the timing diagram and the overall interactions between sub-agents.

The controller, through the previously mentioned communications paths, informs whether they will be served or not during the current green phase. This is possible as given in Equation 4 above and the green time for the designated approach. Immediate benefit from this is embedded speed control for vehicles not in the queue. When a driver knows that it is hard to catch the traffic signal green, he/she keep his speed normal and does not step on the gas.

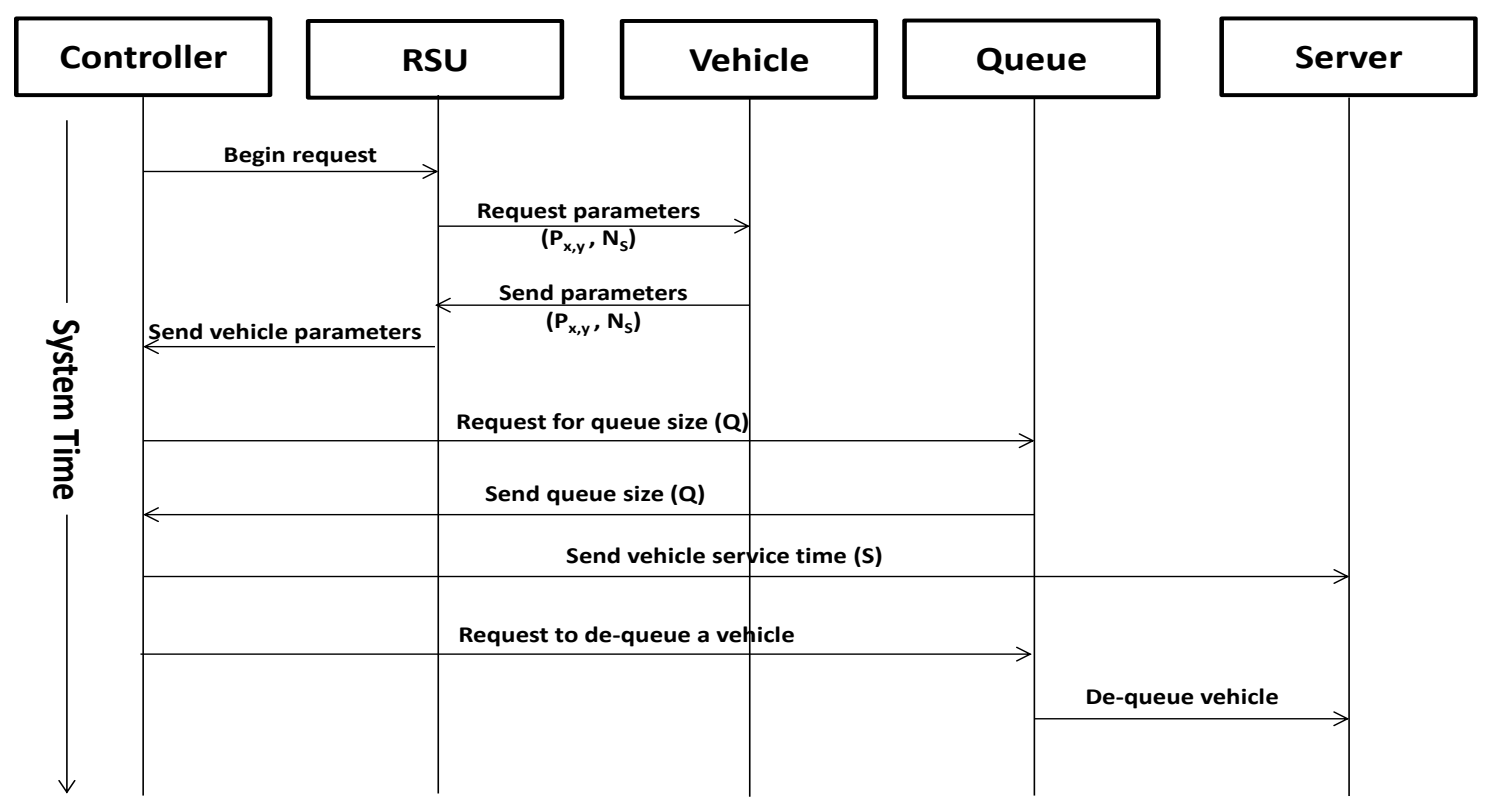

Figure 3 Timing diagram for the multi-agent system and the interaction between sub-agents

\section{Discrete event model description}

Matlab/Simulink/SimEvents was used in this research to develop a discrete event model. SimEvents is a discrete-event simulation engine and component libraries. It is a tool for analyzing event-driven system models and optimizing performance characteristics. SimEvents enables model routing, processing delays, and prioritization for scheduling and communication.

The block diagram of Simulink model is shown in Figure 4. The traffic signal is modelled as an input switch with inputs equals to the number of roads at the intersection, i.e. IN1 to IN4. The controller block represents the traffic signal controller. It controls the switching time based on the traffic densities for all four roads and the cycle time, which represents the total time of one cycle of the traffic signal. The cycle is assumed equal to the green times of the four phases plus four yellow times. The output of the controller is the port number of the input switch. The green time for each phase is calculated as a portion of the cycle time based on the traffic signal of that phase. The service time subsystem is used to model the delay time of the vehicle while crossing the traffic signal. The maximum and minimum service times are assumed four and two seconds respectively. The maximum service time is for the first vehicle in the queue. Then service time declines for the preceding vehicle until reach the minimum. The phase subsystems, i.e. Phase1 to Phase4, generates a number of vehicles and their speed randomly at each phase (road) as detailed in Figure 5. It consists of an entity generator where entities are generated upon a port connected to an event-based random number. Inter-arrival time is modelled as an exponentially distributed random variable. A set-attribute block is used to set speed attribute for each entity. Speed is assumed to be a random variable that uniformly distributed between 20 and $60 \mathrm{~km} / \mathrm{h}$. The delay time between the entity generator and the traffic signal depends on the speed of the entity. The delay time is modelled by an infinite server where the service time is the delay time. The delay time is calculated from the distance and the speed by dividing the distance by the speed. The traffic density which represents the number of vehicles in one-kilometer length of the 
Ahmad Aljaafreh et al.

street equals to the number of entities delayed in the infinite server block.

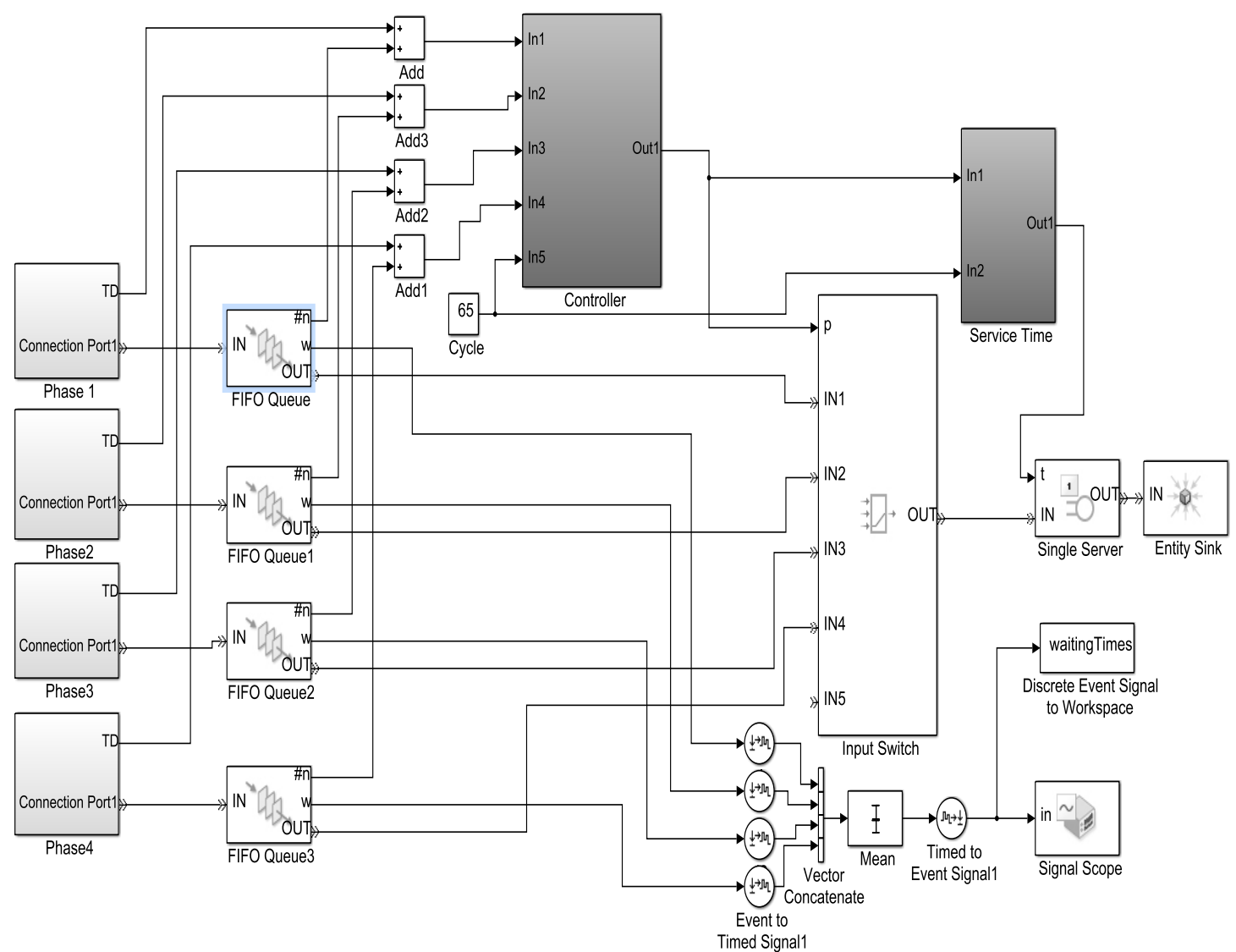

Figure 4 Block diagram for top-level Simulink model of four-way intersection system. The traffic signal is modelled as an input switch with four roads input; Phase1 to Phase4 represent vehicles generated at each road; the switching time for each road is controlled by the controller; Service time represents the delay time of the vehicle while crossing the traffic signal

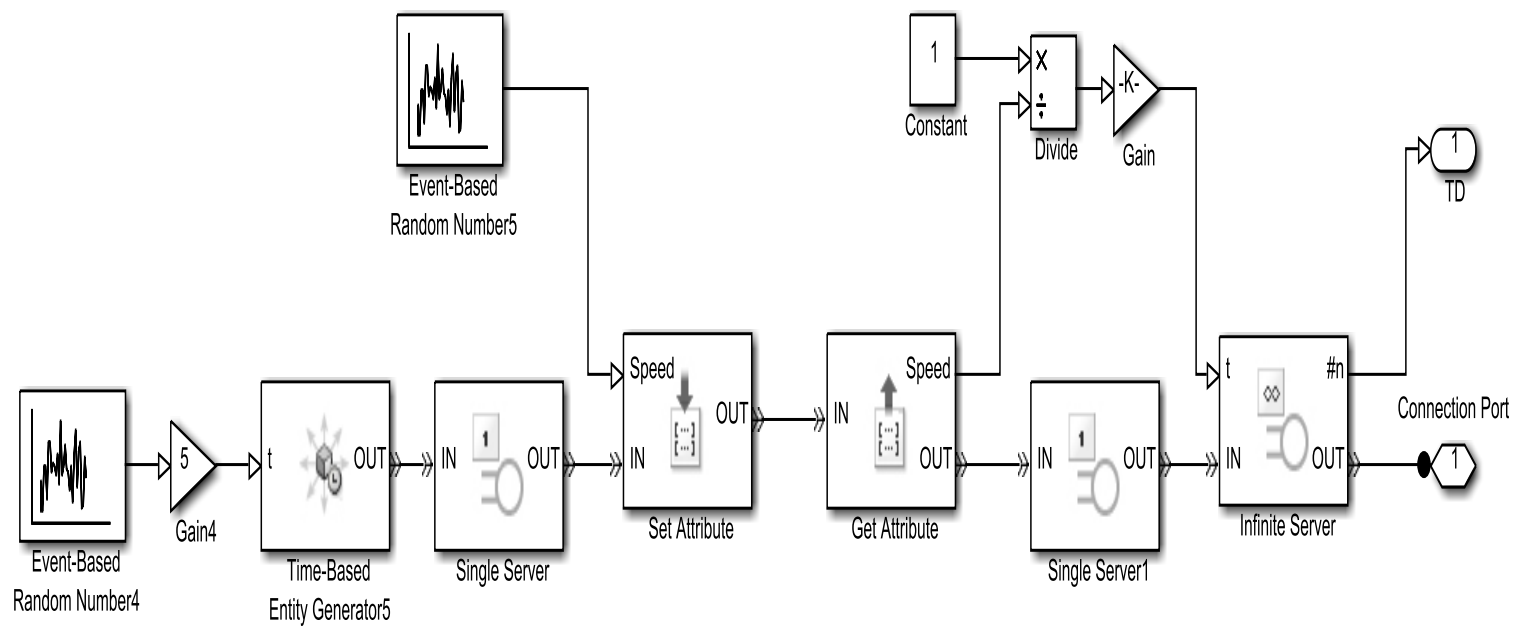

Figure 5 Phase subsystem diagram 


\section{Simulation results and discussion}

\subsection{Simulation parameters}

The proposed algorithms were assessed using extensive simulation experiments. 50 runs using Independent and identically distributed (IID) random variables for 10000 simulation time units were conducted. The yellow time $\left(T_{y}\right)$ is set to 4 seconds. The speed of the vehicles is random variable that uniformly distributed between 20 and $60 \mathrm{~km} / \mathrm{h}$. The length of the road segment is considered to be $1 \mathrm{Km}$.

\subsection{Model verification}

Our proposed model was verified by intensive simulations using four main parameters: (1) green phase; (2) inter-arrival times; (3) service time; and (4) average waiting time $(A W T)$.

4.2.1Model verification using the green phase

In this simulation, two cycles of 65 seconds length each were generated by the model with zero-vehicle generation as shown in Figure 6.

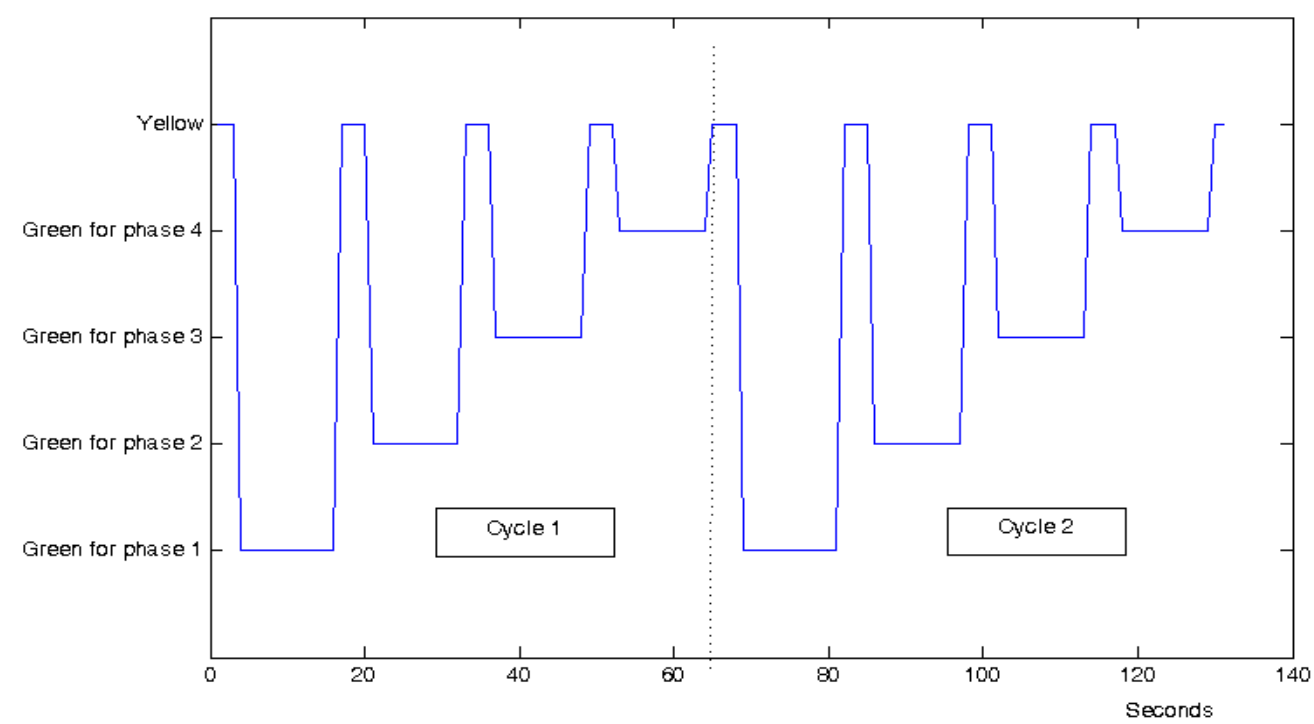

Figure 6 Green phases for the four road approaches of the traffic signal during two cycles of 65 seconds. The green phases are equal for the invariant-traffic generation

Figure 6 shows that the green phases are equal for the 4-road approaches used in our system. The green times are equal, as expected, since there is no variance in the vehicle density and arrival rate. Such equality verified the model, where the main cause is the invariant-traffic generated by our proposed model.

4.2.2Model verification using the inter-arrival times In this simulation, we monitor the generated interarrival times of the vehicle nodes for the four road approaches by running the proposed system for 250 seconds. Figure 7 provides a verification of the proposed model, where it shows the variability of the inter-arrival times as the simulation time goes by.
4.2.3Model verification using the service time In this simulation, two cycles of 65 -second length each were generated by the model with zero-vehicle generation as shown in Figure 8.

Figure 8 provides a verification of the proposed system through monitoring the service time for each approach, where it depicts the randomness in such system parameter. It shows that in each green phase the generated service times are random and uniformly distributed between $[0.5,2]$ seconds.

4.2.4Model verification using the average waiting time $(A W T)$ :

In this simulation, the model is verified using $A W T$ metric. $A W T$ is calculated for distinct inter-arrival time (IAT) averages. The simulation results are shown in Table 1. 


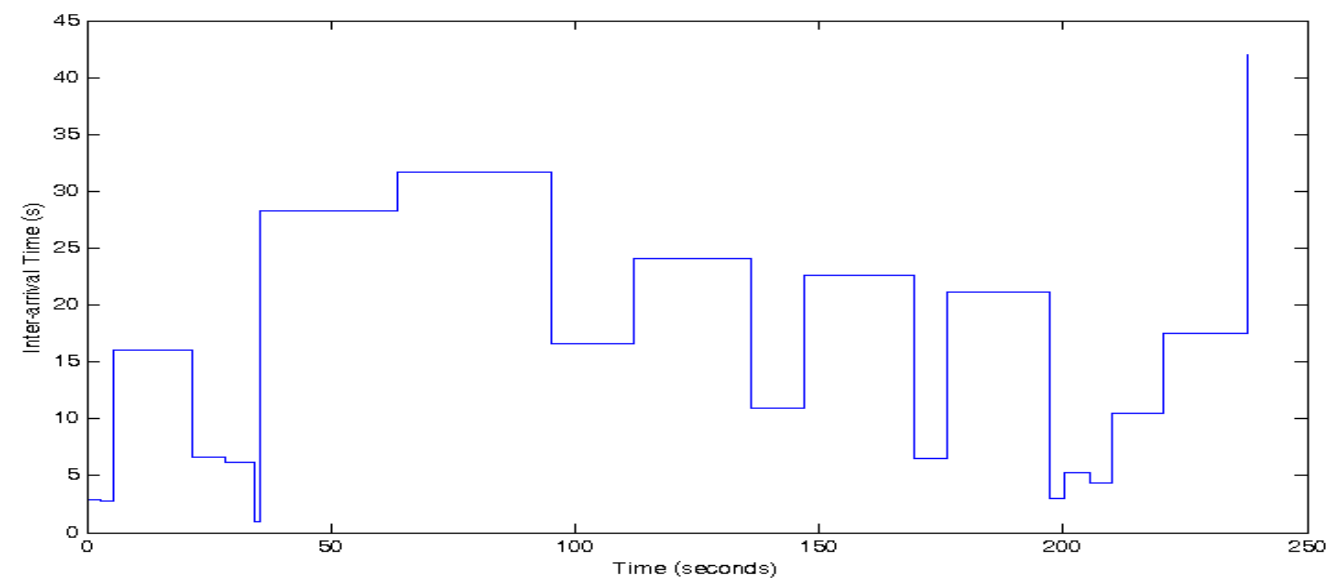

Figure 7 Inter-arrival times for the four road approaches vs. simulation time

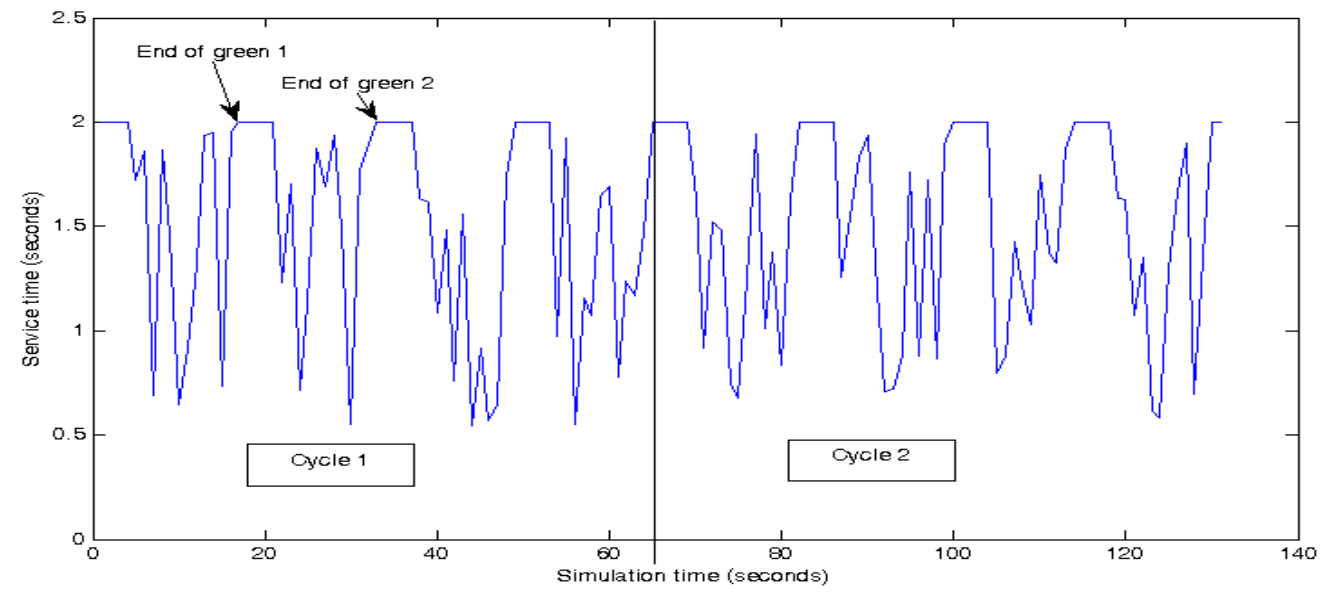

Figure 8 Service times during the first two cycles in each green phase

Table 1The average waiting times (AWT) of vehicles generated at distinct inter-arrival time (IAT) means for 65and 80-seconds cycles

\begin{tabular}{lll}
\hline IAT mean (S) & AWT (TD+Q) & AWT (TD+Q) \\
& Cycle 65 S & Cycle 80 S \\
\hline 35 & 24 & 26 \\
30 & 24 & 26 \\
25 & 26 & 26 \\
20 & 29 & 30 \\
15 & 43 & 40 \\
\hline
\end{tabular}

The first column, in the Table, is the mean of the exponentially distributed inter-arrival time for vehicles generation, the mean is the same for the four road approaches. The average waiting time is calculated for two different cycles of 65 and 80 seconds. It is apparent that the average waiting time increases as the mean of inter-arrival time of vehicle generation decreases, and thus the generated model is verified.
4.3Impact of the average waiting time:

In this simulation, the performance of the proposed adaptive controller $(T D+Q)$ was measured in comparison with a fixed time controller that uses a preset green time based on a prior knowledge of the traffic flow. The performance metric was the average waiting time for all approaches at a traffic signal. The simulation was performed by measuring such metric for different variances of the vehicles, inter-arrival times in the range $[0,130]$. The simulation results 
shown in Figure 9 illustrated that the adaptive controller outperforms the fixed-time controller when there is a significant variance (i.e. variance $>10$ ) in the means of inter-arrival times for vehicles approaching the traffic signal, whereas the fixed-time outperforms the adaptive controller when the variance of the means of the inter-arrival times is less than 10 .
In order to find the optimal cycle time for the proposed system, the simulations were performed by measuring the average waiting time for several interarrival time variances and the cycle time. The simulation results indicated that the optimal cycle time is around 50 seconds regardless of the interarrival time variance, as shown in Figure 10.

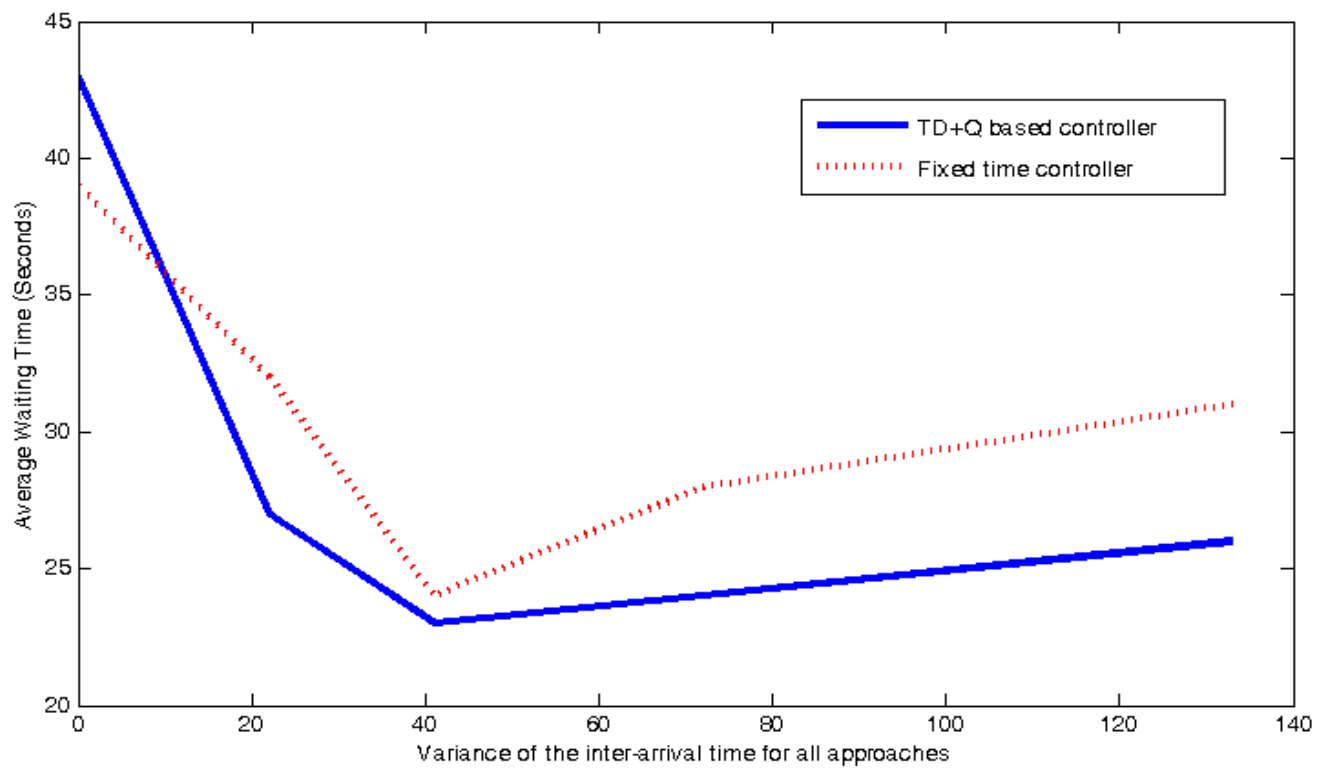

Figure 9 The average waiting times vs. the inter-arrival times variance for an adaptive and fixed-time controller

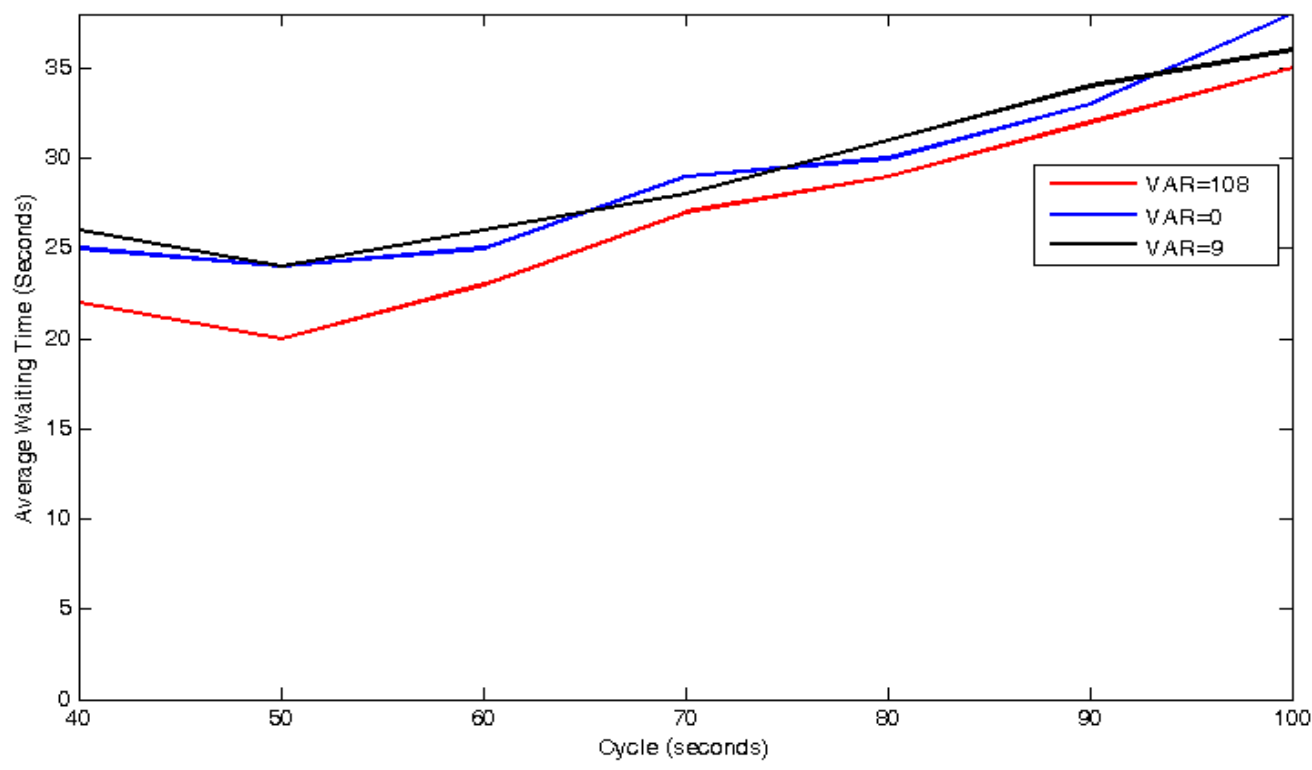

Figure 10 The average waiting time for several inter-arrival time variances vs. cycle time 


\section{Conclusions and future work}

In this study, a model for an adaptive traffic density controller (TD + Q) was developed and verified for different traffic density parameters. The average waiting time (AWT) of the proposed model was then compared to a fixed-time baseline controller. The simulation results revealed that the proposed controller outperformed the baseline controller for variant inter-arrival times due to its adaptability to the variations in input parameters. The variance impact of the inter-arrival times was investigated for both controllers. It has been shown that the proposed controller outperformed the baseline one as the variance of inter-arrival times increased.

The advantage of the fixed-time controller compared to the adaptive one is the simplicity of the controller. However, it does not adapt to variable traffic situations which degrades the performance. The only limitation of applying the proposed model is the assumption of inter-vehicles communication for reporting the position and the speed of the vehicles. This limitation can be overridden through the process of virtual road segmentation and the deployment of road-side units (RSUs) for communication.

As a future work, the cycle length and the sequence of phases will be considered as adapted parameters. Another future direction is to consider more than one intersection as a distributed control system.

\section{Acknowledgment}

None.

\section{Conflicts of interest}

The authors have no conflicts of interest to declare.

\section{References}

[1] Zheng X, Recker W, Chu L. Optimization of control parameters for adaptive traffic-actuated signal control. Journal of Intelligent Transportation Systems. 2010; 14(2):95-108.

[2] Yousef KM, Al-Karaki MN, Shatnawi AM. Intelligent traffic light flow control system using wireless sensors networks. Journal of Information Science and Engineering. 2010; 26(3):753-68.

[3] Sumaryo S, Halim A, Ramli K. Improved discrete event simulation model of traffic light control on a single intersection. In international conference on QiR 2013 (pp. 116-20). IEEE.

[4] Soh AC, Rhung LG, Sarkan HM. MATLAB simulation of fuzzy traffic controller for multilane isolated intersection. International Journal on Computer Science and Engineering. 2010; 2(4):92433.
[5] Tafila J. Security aware routing protocol for intelligent transportation distributed multi-agent system. International Journal of Computer Applications.2018; 180(10):5-13.

[6] Saleh ME, Aljaafreh A, Al-Oudat N. Hierarchal scheduling algorithm for congestion traffic control using multi-agent systems. International Journal of Advanced Computer Research. 2014; 4(17):915-21.

[7] Olfati-Saber R, Fax JA, Murray RM. Consensus and cooperation in networked multi-agent systems. Proceedings of the IEEE. 2007; 95(1):215-33.

[8] Mwangi CM, Kang'ethe SM, Nyakoe GN. Design and simulation of a fuzzy logic traffic signal controller for a signalized intersection. In scientific conference proceedings 2012 (pp. 210-9).

[9] Liu CL, Layland JW. Scheduling algorithms for multiprogramming in a hard-real-time environment. Journal of the ACM. 1973; 20(1):46-61.

[10] Khalid M. Intelligent traffic lights control by fuzzy logic. Malaysian Journal of Computer Science. 1996; 9(2):29-35

[11] Astarita V, Giofrè VP, Guido G, Vitale A. A single intersection cooperative-competitive paradigm in real time traffic signal settings based on floating car data. Energies. 2019; 12(3):1-22.

[12] Yao Z, Shen L, Liu R, Jiang Y, Yang X. A dynamic predictive traffic signal control framework in a crosssectional vehicle infrastructure integration environment. IEEE Transactions on Intelligent Transportation Systems. 2019.

[13] Zhao Y, Ioannou P. A co-simulation, optimization, control approach for traffic light control with truck priority. Annual Reviews in Control. 2019; 48:283-91.

[14] Gershenson C. Self-organizing traffic lights. arXiv preprint nlin/0411066. 2004.

[15] George AM, Palika Shetty S. Fuzzy controller for an image based traffic system. International Journal of Management, IT and Engineering. 2012; 2(6):291305.

[16] El-Tantawy S, Abdulhai B, Abdelgawad $\mathrm{H}$. Multiagent reinforcement learning for integrated network of adaptive traffic signal controllers (MARLIN-ATSC): methodology and large-scale application on downtown Toronto. IEEE Transactions on Intelligent Transportation Systems. 2013; 14(3):1140-50.

[17] Azimirad E, Pariz N, Sistani MB. A novel fuzzy model and control of single intersection at urban traffic network. IEEE Systems Journal. 2010; 4(1):107-11.

[18] Aljaafreh A, Al Oudat N. Optimized timing parameters for real-time adaptive traffic signal controller. In UKSim-AMSS 16th international conference on computer modelling and simulation 2014 (pp. 244-7). IEEE.

[19] Aljaafreh A, Al-Oudat N, Saleh ME. Adaptive trafficsignal control using discrete event simulation model. International Journal of Computer Applications. 2014; 101(12):7-12. 


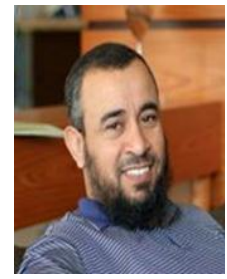

Dr. Ahmad Aljaafreh received his $\mathrm{PhD}$ in Computer Engineering in 2010 from Western Michigan University. In the same year, he joined Tafila Technical University in Tafila, Jordan. Currently, he is an Associate Professor in the Department of Communications, Electronics and Computer Engineering at Tafila Technical University. His research interests are AI and Embedded Systems.

Email: a.aljaafreh@ttu.edu.jo

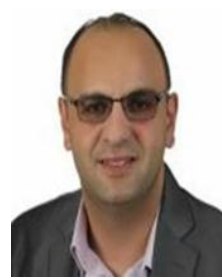

Dr. Ma'en Saleh (M'10) received his Ph.D. degree in Electrical and Computer Engineering from Western Michigan University in 2012. He joined the faculty of Tafila Technical University as an Assistant Professor of Electrical and Computer Engineering in 2012. He promoted to Associate Professor in 2018. His research interests include Real-Time Scheduling for Packet Switched Networks, Security in VANETs, Simulating Real-Time Networks, Real-Time Agent-Based Systems, and QoS for Heterogeneous Networks.

Email: maen@ttu.edu.jo

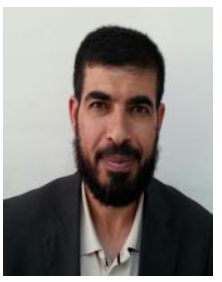

Dr. Naeem Al-Oudat received his PhD in Computer Engineering form Iowa State University in 2012. Since 2012, he has been serving as a faculty member in Communications and Computer Engineering Department at Tafila Technical University. His research interests include Real-Time Distributed Systems Task Optimizing and Scheduling, Educational Operating Systems Design.

Email: naeemodat@ttu.edu.jo

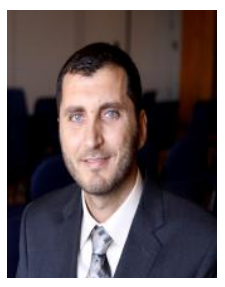

Dr. Murad Alaqtash is an Assistant Professor of Computer Engineering at Tafila Technical University. He received his $\mathrm{PhD}$ degree in Computer Engineering from the University of Texas at El Paso, Texas, USA in 2012. His research interests are Artificial Intelligence, Machine Learning, Smart Embedded Systems, and Wearable Sensors.

Email: mmalaqtash@ttu.edu.jo 